\title{
Hyperkinetic motor seizures: a common semiology generated by two different cortical seizure origins
}

\author{
Lisa Vaugier ${ }^{1}$, Aileen McGonigal ${ }^{1,2}$, Stanislas Lagarde ${ }^{1,2}$, \\ Agnes Trébuchon ${ }^{1,2}$, William Szurhaj ${ }^{3}$, Philippe Derambure ${ }^{3}$, \\ Fabrice Bartolomei ${ }^{1,2}$ \\ ${ }^{1}$ APHM, Hôpital de la Timone, Service de Neurophysiologie Clinique, Marseille \\ ${ }^{2}$ Aix-Marseille Univ, INSERM U751, Marseille \\ ${ }^{3}$ Université de Lille II, Lille, Nord-Pas-de-Calais, France
}

Received November 23, 2016; Accepted June 16, 2017

ABSTRACT - We report a 37-year-old, right-handed patient with drugresistant focal epilepsy whose seizures were characterized by explosive hyperkinetic behaviour. Video-SEEG revealed bifocal organization of epilepsy with two distinct cortical origins of seizures: the right temporal pole and left temporal lateral and perisylvian cortex. Irrespective of the cortical pattern of seizure onset, the hyperkinetic semiology was extremely similar. This supports a major role for "final common pathway" subcortical circuits in the genesis of the hyperkinetic semiology in this patient.

Key words: focal epilepsy, hyperkinetic / hypermotor semiology, central pattern generator

Seizures involving hyperkinetic (or hypermotor) behaviour are characterized by excessive and sometimes explosive movements (Blume et al., 2001), the pathophysiological basis of which remains poorly understood. While hyperkinetic semiological patterns are diverse, their common feature is excessive motor activity, predominant in axial and proximal structures (Rheims et al., 2008; Bonini et al., 2014). Patients may present with large movements, usually rapid balletic limb movements, which may be of variable directionality and rhythmicity, often associated with axial movements such as trunk torsion or pelvic thrusting. The excessive motor activity may be associated or not with emotional motor expression such as fear or anger (appearance suggestive of attack or defence), in which case vocalization is a common feature; the apparent emotional context and recognisable pattern of behaviour lends an "integrated" appearance to the motor signs (Bonini et al., 2014). In other semiological patterns, the excess motor activity may appear organised in complex motor sequences, resembling physiological activity such as cycling, walking or boxing (Nobili et al., 2004; Tassinari et al., 2005a; Nobili et al., 2007). Because of their 
excessively repetitive nature, such ictal motor patterns can be also characterized as stereotypies (McGonigal et al., 2008). Other seizures with hyperkinetic movements show neither emotional expression nor clearly recognisable physiological-type movement sequences, but rather apparent uncoordinated and disjointed movements, which may be associated with tonic and sometimes dystonic limb and/or facial contraction (Bonini et al., 2014).

Explosive hyperkinetic motor behaviour was first described in the context of orbitofrontal seizures (Tharp, 1972) and was subsequently recognized as a typical component of nocturnal frontal seizures (Rheims et al., 2008; Bonini et al., 2014). However, over the past decade, the concept of a univocal frontal origin has been challenged by several reports showing that other cortical epileptogenic zones (such as the temporal pole and parieto-insular region) may trigger similar hyperkinetic behaviour during seizures (Nobili et al., 2004; Ryvlin et al., 2006; Vaugier et al., 2009). Of course, seizures with semiology characterized by complex motor behaviour tend to involve widely distributed cortical networks, and not only seizure onset but propagation patterns are key to clinical expression (Bartolomei et al., 2013). The hypothesis that predominantly subcortical generators lead to some hyperkinetic behaviours has been postulated, with the idea that loss of higher cortical control during the seizure leads to release of "instinctive motor behaviours" (Tassinari et al., 2005a).

Here, we report a patient with nocturnal hyperkinetic seizures, in whom essentially the same semiological expression was produced by seizure origins from either the right temporal polar cortex or the left perisylvian region. This case adds further evidence to the hypothesis that some forms of hyperkinetic semiology are not directly produced by a specific cortical region but are more dependent upon subcortical organization.

\section{Case presentation}

A 37-year-old, right-handed man was admitted for assessment of intractable refractory epilepsy. Birth and psychomotor development were normal. He had complex febrile seizures at 9 months. Seizures started at age 13 years and were mostly nocturnal with hyperkinetic semiology. He was sometimes able to report a subjective experience of a rising unpleasant warm sensation in the body, associated with dyspnoea. He would then present facial grimacing and fairly explosive hyperkinetic motor signs with pedalling, side-to-side semi-rhythmic rocking, rotation of the body, neck extension, and pelvic thrusting. Seizures were resistant to antiepileptic drugs and recurred every night. Long- term video-EEG showed interictal right anterior spikes and sharp waves. Ictal video-EEG showed semiology as described above, with bilateral flattening on scalp EEG. Cerebral MRI was normal and FDG-PET (positron emission tomography) showed left temporo-polar and left fronto-opercular hypometabolism. Stereoelectroencephalography (SEEG) was indicated with the aim of identifying the epileptogenic zone. SEEG exploration was carried out during long-term video-EEG monitoring. Recordings were performed using intracerebral multiple-contact electrodes (10-15 contacts; length: $2 \mathrm{~mm}$; diameter: $0.8 \mathrm{~mm} ; 1.5 \mathrm{~mm}$ apart), placed according to Talairach's stereotactic method, as previously reported (Bartolomei et al., 2008). Signals were recorded on a 128-channel Deltamed TM system. They were sampled at $256 \mathrm{~Hz}$ and recorded on a hard disk (16 bits/sample), without a digital filter. The SEEG scheme is indicated in figure 1. Two separate epileptogenic zones were identified, associated with very similar hyperkinetic semiological expression (figure 2): one with seizure onset in the right temporal pole/orbitofrontal cortex system (figure 1A) and the other in the left temporal lateral cortex and opercular region (figure 1B). The right temporo-polar seizures were characterized by rapid changes in facial expression (with grimace more pronounced on the right side) and significant hyperkinetic semiology including repetitive rocking and rotation of the body (figure 2). The first electrical changes were observed under the form of a rapid discharge affecting the temporal pole, preceded by a polyspike complex (figure 1A). No involvement of the cingulate gyrus was observed. The left operculo-insular seizures were sometimes associated with an aura (disgust and throat striction). Ictal symptoms included first swallowing movements and a slightly more delayed hyperkinetic phase. Hyperkinetic movements were very similar (prominent rotational movement and facial contortion) (figure $2 B$ ). The initial SEEG discharge was observed in the left posterior insular cortex under the form of a rhythmic discharge with beta frequency (figure 1B). A delayed involvement of the frontal opercular regions, the anterior insular cortex, and the temporal regions was observed.

The bilateral/multifocal organization of the epilepsy finally led to contraindication for curative surgery.

\section{Discussion}

This case shows that two distinct, independent and bilateral epileptogenic cortical networks may produce almost identical hyperkinetic seizure semiology in the same patient. The specific epileptogenic cortical regions involved in our case (the left operculoinsular and temporal polar-orbitofrontal regions) have 


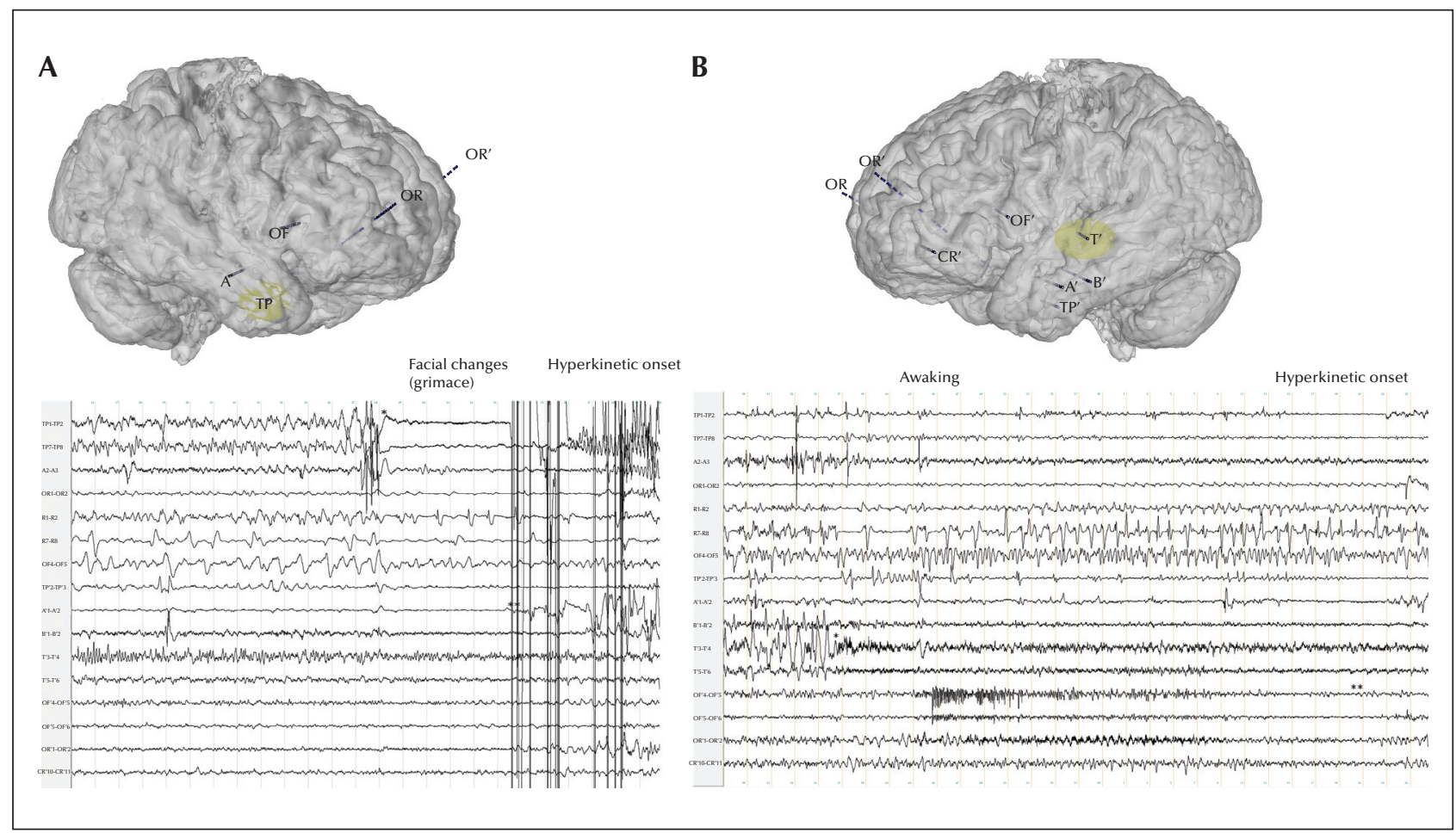

Figure 1. SEEG implantation and the two different ictal onsets recorded in the patient. (A) Seizure starts from the right temporal polar cortex by a rapid discharge $\left(^{*}\right)$ before the emergence of clinical semiology. (B) Seizure starting from the left temporal region (firstly from the temporal gyrus) and then the fronto-opercular region. The pattern here is different with changes first appearing at the $\mathrm{T}^{\prime}$ electrodes $(*)$ and then involving the left orbito-frontal region $\left(\mathrm{OR}^{\prime},{ }^{* *}\right)$ and the left opercular cortex $\left(\mathrm{OF}^{\prime}\right)$. The ictal hyperkinetic sequence is delayed following seizure onset.

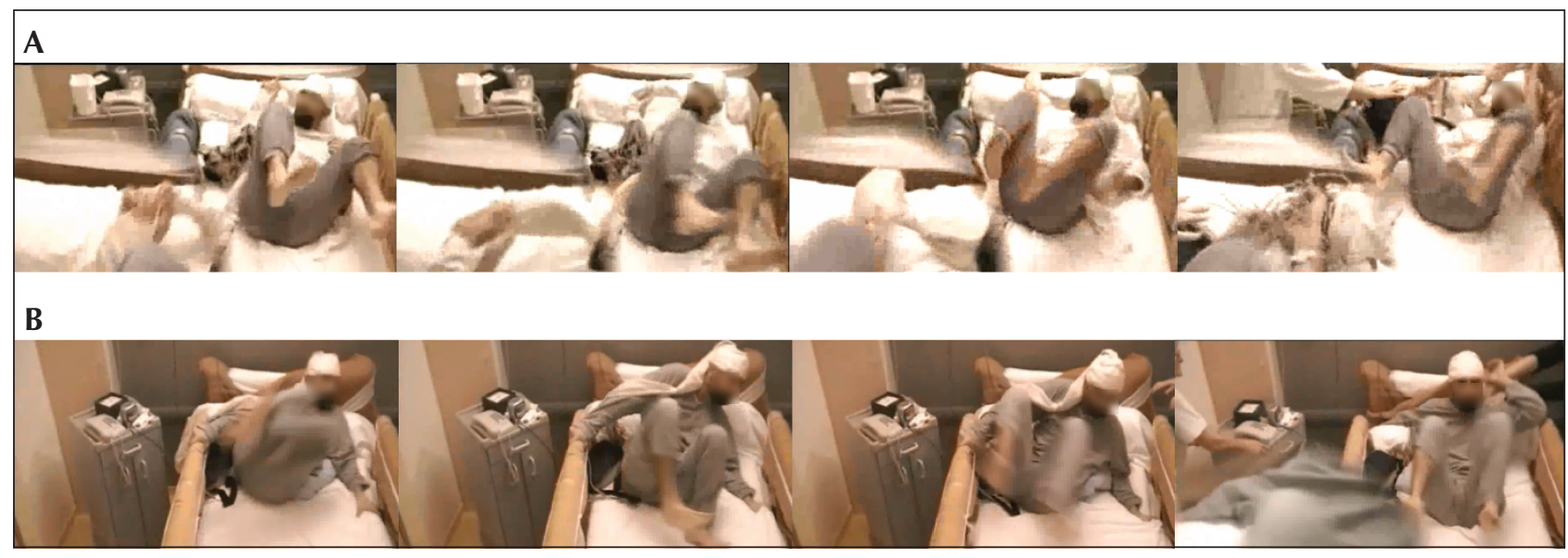

Figure 2. Sequences of hyperkinetic signs for the two types of seizures (lasting for 30 seconds) with (A) right temporo-polar onset and (B) left temporo-perisylvian onset.

previously been identified as a potential source of hyperkinetic seizures. In particular, temporo-frontal seizures (with preferential involvement of the temporal pole and orbital frontal cortex) (Nobili et al., 2004; Vaugier et al., 2009) have been reported with ictal hyperkinetic motor behaviour. Hyperkinetic motor behaviour encompasses many diverse semiological presentations which seem likely to have different pathophysiological mechanisms. As such, the heterogeneous category "hyperkinetic seizures" per se is not necessarily a useful one when trying to elucidate the brain dynamics involved. For example, 
hyperkinetic motor behaviour, including intense emotional expression (screaming, fearful and/or angry facial expression, swearing, or attack behaviour), has been well-described in seizures involving the limbic fronto-temporal system (Bartolomei et al., 2005; Tassinari et al., 2005b; Bonini et al., 2014). In this context, emergence of explosive motor behaviour has been related to decoupling between the orbitofrontal cortex and the amygdala (key structures within the emotional limbic system), directly produced by altered network dynamics due to cortical seizure activity, which is postulated to produce dysregulation of cortical emotional control (Bartolomei et al., 2005). The repetitive nature of some hyperkinetic behaviour (such as rhythmic body rocking) suggests the possibility of a similar mechanism to that involved in the production of motor stereotypies which may occur in other clinical conditions, involving cortical-striatal circuits (Edwards et al., 2012). In the context of epilepsy, recognisable motor patterns, especially locomotor behaviour but also grooming or eating behaviour, have been interpreted to be related to the release of primitive, stereotyped motor behaviours, depending on central pattern generators, which can also be observed in some sleep disorders (Tassinari et al., 2005a). However, possible mechanisms and the circuitry involved remain unknown, mainly because electrophysiological investigation of such epilepsy cases predominantly involves only the cortical components of the likely involved cortico-subcortical circuits, based on clinical indication. It is probable that cortico-subcortical interactions, depending on the precise localization and electrophysiological pattern of cortical seizure activity, can influence clinical expression. There is some evidence for the latter in terms of patterns of motor stereotypies in frontal seizures, which seem to be organized along a rostro-caudal cortical axis (McGonigal and Chauvel, 2014). While intracranial EEG offers relatively limited possibilities for better understanding the role of subcortical structures, functional imaging may provide some clues, although ictal recordings are rare. In an interictal PET study, significant hypometabolism was observed in the caudate head and midbrain in patients showing ictal hyperkinetic behaviour, compared to those without (Guedj et al., 2012). In another study, Masuda and co-workers have used ictal SPECT and voxel-based analysis to identify the neuronal networks generating specific ictal symptomatology in patients with hyperkinetic seizures. They showed that both cortical and subcortical regions are involved during these kinds of seizures. In particular, the mesencephalic locomotor nucleus could be the most likely relay to trigger access to spinal central pattern generators responsible for the hyperkinetic movements (Masuda et al., 2012).
Our case adds further indirect evidence for the crucial role of subcortical organization of some forms of hyperkinetic motor behaviour. While subcortical circuits necessarily play an important part of any semiological expression, it may be that some semiological patterns are more "cortical-dependent" whereas others are more "subcortical-dependent". The present observation is indeed in favour of this hypothesis; since essentially identical hyperkinetic behaviour could emerge from seizure onset in two distinct and bilaterally independent cortical networks, it seems likely that this was expressed via a "final common pathway", organized within basal ganglia and brainstem structures.

\section{Disclosures.}

The authors have no conflict of interest to disclose.

\section{References}

Bartolomei F, Trebuchon A, Gavaret M, Regis J, Wendling F, Chauvel P. Acute alteration of emotional behaviour in epileptic seizures is related to transient desynchrony in emotion-regulation networks. Clin Neurophysiol 2005; 116: 2473-9.

Bartolomei F, Chauvel P, Wendling F. Epileptogenicity of brain structures in human temporal lobe epilepsy: a quantified study from intracerebral EEG. Brain 2008; 131: 1818-30.

Bartolomei F, Guye M, Wendling F. Abnormal binding and disruption in large scale networks involved in human partial seizures. EPJ Nonlinear Biomed Phys 2013; 1.

Blume WT, Luders HO, Mizrahi E, Tassinari C, van Emde Boas W, Engel J Jr. Glossary of descriptive terminology for ictal semiology: report of the ILAE task force on classification and terminology. Epilepsia 2001; 42: 1212-8.

Bonini F, McGonigal A, Trebuchon A, et al. Frontal lobe seizures: from clinical semiology to localization. Epilepsia 2014; 55: 264-77.

Edwards MJ, Lang AE, Bhatia KP. Stereotypies: a critical appraisal and suggestion of a clinically useful definition. Mov Disord 2012; 27: 179-85.

Guedj E, McGonigal A, Vaugier L, Mundler O, Bartolomei F. Metabolic brain PET pattern underlying hyperkinetic seizures. Epilepsy Res 2012; 101: 237-45.

Masuda H, Shariff E, Tohyama J, Murakami H, Kameyama S. Clinical patterns and pathophysiology of hypermotor seizures: an ictal SPECT study. Epileptic Disord 2012; 14:32-40.

McGonigal A, Chauvel P. Prefrontal seizures manifesting as motor stereotypies. Mov Disord 2014; 29:1181-5.

McGonigal A, Gavaret M, Da Fonseca AT, et al. MRI-negative prefrontal epilepsy due to cortical dysplasia explored by stereoelectroencephalography (SEEG). Epileptic Disord 2008; 10:330-8. 
Nobili L, Cossu M, Mai R, et al. Sleep-related hyperkinetic seizures of temporal lobe origin. Neurology 2004; 62: 482-5.

Nobili L, Francione S, Mai R, et al. Surgical treatment of drugresistant nocturnal frontal lobe epilepsy. Brain 2007; 130:56173.

Rheims S, Ryvlin P, Scherer C, et al. Analysis of clinical patterns and underlying epileptogenic zones of hypermotor seizures. Epilepsia 2008; 49(12): 2030-40.

Ryvlin P, Minotti L, Demarquay G, et al. Nocturnal hypermotor seizures, suggesting frontal lobe epilepsy, can originate in the insula. Epilepsia 2006; 47: 755-65.
Tassinari CA, Rubboli G, Gardella E, et al. Central pattern generators for a common semiology in fronto-limbic seizures and in parasomnias. A neuroethologic approach. Neurol Sci 2005a; 26(3): s225-32.

Tassinari CA, Tassi L, Calandra-Buonaura G, et al. Biting behavior, aggression, and seizures. Epilepsia 2005b; 46: 654-63.

Tharp BR. Orbital frontal seizures. An unique electroencephalographic and clinical syndrome. Epilepsia 1972;13: 627-42.

Vaugier L, Aubert S, McGonigal A, et al. Neural networks underlying hyperkinetic seizures of "temporal lobe" origin. Epilepsy Res 2009; 86: 200-8. 